\title{
Conocimiento de la estadística y los estudiantes en futuros profesores: un estudio exploratorio
}

\author{
Pedro Arteaga, Universidad de Granada (España) \\ J. Miguel Contreras, Universidad de Granada (España) \\ Gustavo R. Cañadas, Universidad de Granada (España)
}

Recibido el 12 de Septiembre de 2014; aceptado el 3 de octubre de 2014

\section{Conocimiento de la estadística y los estudiantes en futuros profesores: un estudio exploratorio}

\section{Resumen}

El objetivo de este trabajo fue evaluar el conocimiento de la estadística y los estudiantes, que una muestra de 108 futuros profesores de Educación Primaria, pone en juego al analizar un proyecto de análisis de datos en el que habían trabajado previamente. Para ello se proporcionó a los participantes la guía de análisis de la idoneidad cognitiva y afectiva propuesta por Godino (2009). El análisis cualitativo de las respuestas permitió definir unos niveles de aplicación de los distintos descriptores propuestos, mostrando los participantes mayor competencia para analizar la idoneidad cognitiva que la afectiva. Se concluye con la necesidad de mejorar el conocimiento de estadística y los estudiantes en los futuros profesores.

Palabras clave. Conocimiento del contenido de estadística y los estudiantes, idoneidad cognitiva, idoneidad afectiva, formación de profesores.

\section{Knowledge of statistics and students in prospective school teachers: an exploratory study}

Abstract

This work intend to analyze the knowledge of statistics and students in a sample of 108 pre-service primary school teachers when analyzing a teaching unit based on a statistical project. We provide the participants with the guide to analyze the cognitive and affective suitability proposed by Godino (2009). The qualitative analysis of responses served to define levels in the application of the different descriptors with better performance as regards cognitive suitability than affective suitability. We conclude the need to improve the knowledge of statistics and students in prospective school teachers. education.

Key words: Knowledge of content and students, cognitive suitability, emotional suitability, teacher

\section{Conhecimentos de estatística e alunos em futuros professores: um estudo exploratório}

\section{Resumo}

O objetivo deste estudo foi avaliar o conhecimento da estatística e dos estudantes, en uma amostra de 108 professores do ensino fundamental, utilizado um projeto de análise de dados em que havian trabalhado anteriormente. Para este fim, os participantes foram fornecidas as orientações para analisar a adequação cognitiva e afetiva dada pelo Godino (2009). A análise qualitativa das respostas Para citar: Arteaga, P, Contreras, J. M. \& Cañadas, G. R. (2014). Conocimiento de la estadística y los estudiantes en futuros profesores: un estudio exploratorio. Avances de Investigación en Educación Matemática, 6, 63 - 84 . 
serviu para definir os níveis de implementação dos vários descritores propostos, mostrando aumento da competencia de participantes para analisar a adequação cognitiva que afetiva. Conclui-se com a necessidade de melhorar o conhecimento de estatística e os alunos em futuros professores.

Palavras chave: Conhecimento del conteúdo e os alunos, adequaçao cognitiva, adequaçao emocional, a formação de professores.

\begin{abstract}
Connaissance de la statistique et des étudiants dans les futurs enseignants: une étude exploratoire.

\section{Résumé}

L'objectif de cette étude est d'évaluer les connaissances de la statistique et des étudiants, d'un échantillon de 108 potential enseignants du primaire, mis en jeu lors de l'analyse d'un projet d'analyse de données dans lequel ils avaient déjà travaillé. Cela a été fourni par l'analyse des participants de la pertinence cognitive et affective definée par Godino (2009). L'analyse qualitative des réponses a permis de définir les niveaux de mise en œuvre des différents descripteurs proposés, indiquant les participants plus de competence pour analyser la pertinence cognitive que l'affective. On conclut à la nécessité d'améliorer la connaissance des statistiques et des étudiants dans les futurs enseignants.
\end{abstract}

Paroles clé: Connaissances des élèves et de la statistique, pertinence cognitive, pertinence émotionnelle, formation des enseignants.

\title{
1. Introducción
}

Hoy día es constante la presencia de la estadística en nuestra sociedad, donde se reconoce su utilidad como una herramienta metodológica que permite analizar la variabilidad, determinar relaciones entre variables, diseñar estudios y experimentos y tomar decisiones adecuadas en situaciones de incertidumbre. El reconocimiento de esta utilidad por las autoridades educativas ha llevado a incorporar la enseñanza de la estadística, en forma generalizada en todos los niveles educativos.

Diferentes directrices curriculares (por ejemplo, NCTM, 2000; CCSSI, 2010) proponen introducir ideas elementales de estadística desde el comienzo de la Educación Primaria. En el caso de España (MEC, 2006), y en este nivel educativo, se ha propuesto un bloque temático denominado Tratamiento de la información, azar y probabilidad en el currículo de Matemáticas. Para el primer ciclo de la Educación Primaria (niños de 6 y 7 años) se sugieren las técnicas elementales para la recogida de datos, los gráficos estadísticos, e introducción al lenguaje del azar. Se continúan estos contenidos en segundo ciclo (8-9 años), incluyendo las tablas de datos y de doble entrada. En tercer ciclo (10-11 años) se amplía el trabajo con gráficos, resaltando la importancia de analizarlos críticamente. Se introduce la media aritmética, moda y rango, y la estimación de la probabilidad de un suceso. Contenidos similares se sugieren en MECD (2014).

En los currículos citados, así como en el proyecto GAISE (Franklin, et al. 2007) se propone adicionalmente una metodología de enseñanza basada en el trabajo con proyectos, mediante los cuales se proporciona a los estudiantes oportunidad para experimentar el ciclo completo de trabajo estadístico. Para ello los estudiantes deben diseñar investigaciones, formulando preguntas de investigación, recoger datos que permitan responder estas preguntas, analizarlos y obtener algunas conclusiones o predicciones sobre las preguntas iniciales, que estén apoyadas en los resultados del análisis de los datos. 
Una condición para asegurar que estas propuestas curriculares para la Educación Primaria se desarrollen adecuadamente es la formación de los profesores de esta etapa educativa, algunos de los cuáles podrían tener carencias formativas o sentirse inseguros al enseñar el tema (Pierce \& Chick, 2011). La razón es que pocas Facultades de Educación proponen un curso completo de estadística y menos aún un curso completo de didáctica de la estadística en sus programas de formación de estos profesores.

La formación adecuada del profesor para enseñar matemáticas preocupa a nivel internacional $\mathrm{y}$, como consecuencia, numerosos investigadores han tratado de conceptualizar la naturaleza del conocimiento profesional del profesor de matemática y describir sus componentes (e.g. las síntesis de Llinares \& Krainer, 2006; Hill, Sleep, Lewis \& Ball, 2007, Wood, 2008, Even \& Ball, 2009 o Dawson, Jaworski \& Wood, 2013).

Respecto a los trabajos centrados en la descripción del conocimiento del profesor para enseñar estadística, Burgess (2006), Batanero y Díaz (2010) y Pinto (2010) destacan la importancia de considerar la naturaleza específica de la estadística en la formación de profesores. Por otro lado, el reciente estudio organizado por la International Commission on Mathematical Instruction (ICMI) y la International Association for Statistical Education (IASE) (Batanero, Burrill \& Reading, 2011) contribuyó a sintetizar la investigación disponible y propuso una extensa agenda de investigación que, progresivamente, está atrayendo a diferentes investigadores.

Uno de los puntos propuestos en dicha agenda, es la evaluación de los conocimientos de los profesores en formación sobre un tema específico de estadística o sobre componentes particulares de su conocimiento. El conocimiento del contenido (en este caso estadística elemental) y los estudiantes es uno de los componentes del modelo que Hill, Ball y Schilling (2008) proponen para analizar el conocimiento del profesor de matemáticas, que se describe en la siguiente sección.

El objetivo de este trabajo fue realizar un estudio exploratorio de evaluación de los conocimientos sobre la estadística elemental y los estudiantes de una muestra de 108 futuros profesores de Educación Primaria. En este trabajo reinterpretamos este componente en el sentido indicado por Godino (2009; 2013) como unión de las facetas cognitivas y afectivas de su modelo del conocimiento del profesor.

Para llevar a cabo este objetivo proponemos a estos estudiantes analizar una experiencia de enseñanza de la estadística, basada en el trabajo con proyectos y experimentada por ellos mismos. Otro propósito es complementar un trabajo anterior (Arteaga, Batanero, Cañadas \& Gea, 2012) en que analizamos el conocimiento especializado de los futuros profesores sobre el trabajo con proyectos en la clase de estadística. Una versión resumida del mismo trabajo fue presentada en Arteaga et al. (2012). A continuación presentamos los fundamentos del estudio, se describe su metodología y se presentan los resultados.

\section{Fundamentos teóricos}

Partimos del modelo del Conocimiento Matemático para la Enseñanza (MKT) (Hill, Schilling \& Ball, 2004; Ball, Thames \& Phelps, 2005; Hill, et al., 2007; Hill, et al., 2008). Los autores lo describen como "el conocimiento matemático que los profesores usan en sus clases para producir instrucción y crecimiento en los estudiantes" (Hill, et al., 2008, p. 347) y lo caracterizan mediante componentes 
relacionadas con el conocimiento del contenido matemático o con el conocimiento didáctico del contenido. Desglosan el conocimiento del contenido matemático en tres subdivisiones:

1. El conocimiento común del contenido (CCK), sería el conocimiento del tema que tiene cualquier estudiante o persona educada; es decir, el necesario por cualquier persona para resolver problemas de naturaleza matemática.

2. El conocimiento especializado del contenido (SCK): es específico del profesor, que lo aplica para proponer tareas matemáticas en un tema particular y articularlas dentro de la enseñanza (Ball, et al., 2005). Incluye, por ejemplo, la capacidad para analizar errores y la evaluación de ideas alternativas, usar distintas representaciones matemáticas y usar de manera explícita el lenguaje matemático.

3. El conocimiento en el horizonte matemático se refiere a aspectos más avanzados que aportan perspectivas al profesor; tiene en cuenta aquellos componentes del tema, no incluidos en el currículo, por ejemplo, sobre la estructura de la disciplina, pero que son útiles para la enseñanza y proporcionan una comprensión más profunda del tema.

Por otro lado, los autores dividen el conocimiento didáctico del contenido en tres componentes:

- El conocimiento del contenido y los estudiantes (KCS) es utilizado por el profesor para enseñar un contenido particular a estudiantes dados: "es el conocimiento de cómo los estudiantes piensan, saben, o aprenden este contenido particular, .... así como las dificultades y concepciones erróneas que podrían tener en el proceso" (Hill, et al., 2008, p. 375). Este será el componente analizado en este trabajo.

- El conocimiento del contenido y la enseñanza (KCT), combina conocimiento con respecto a la enseñanza y conocimiento matemático. Es el conocimiento sobre procesos adecuados para enseñar y evaluar un tema teniendo en cuenta aspectos como elección de ejemplos, ventajas y desventajas de utilizar una u otra representación (Ball, et al., 2005).

- El conocimiento del currículo se refiere a las características del currículo en los diferentes ciclos formativos y le permite al profesor articular sus temas con los de otras asignaturas o áreas de la matemática (Hill, et al., 2008).

Este modelo ha sido adaptado por diversos investigadores para ser utilizado en investigaciones particulares. Para este trabajo, nosotros tomamos la interpretación ampliada de Godino (2009; 2013), que tiene en cuenta seis dimensiones: epistemológica, cognitiva, afectiva, interaccional, mediacional y ecológica, en el conocimiento del profesor de matemáticas. Cada una de estas dimensiones indica el conocimiento que permite al profesor analizar adecuadamente los correspondientes componentes de la idoneidad didáctica de procesos de enseñanza y aprendizaje de las matemáticas en relación a unos estudiantes y un contexto particular.

El concepto de idoneidad didáctica, junto con sus diferentes componentes e indicadores fue introducido por Godino, Contreras y Font (2006) como una guía de 
análisis que permite evaluar y mejorar la enseñanza de las matemáticas y la formación de profesores, pasando de una didáctica descriptiva a otra normativa. Incluye los siguientes componentes (Godino, 2013; Godino, Batanero, Rivas, \& Arteaga, 2013):

- Idoneidad epistémica: Se trata de ver si un proceso de enseñanza refleja adecuadamente el contenido matemático que se pretende enseñar, o bien es incompleto o sesgado. Es decir, si existe una representatividad de los significados institucionales implementados (o pretendidos) en el proceso de estudio, respecto de un significado de referencia, que puede venir dado, por ejemplo, por las directrices curriculares. No se reduce a los componentes conceptual y procedimental considerados en otros marcos teóricos, sino tienen en cuenta asimismo los campos de problemas abordados, el lenguaje y la argumentación.

- Idoneidad cognitiva: Valora el grado en que los contenidos matemáticos pretendidos/ implementados son asequibles a los estudiantes a los que se dirigen, y también hasta qué punto se ha producido el aprendizaje pretendido por el profesor. Godino (2013) indica que la idoneidad cognitiva de un proceso de estudio implica que los contenidos trabajados se encuentran en la zona de desarrollo potencial de los estudiantes (en el sentido de Vygotski, 1934).

- Idoneidad afectiva: Cualquier actividad propuesta a los estudiantes implica no sólo unos conocimientos puestos en juego, sino que moviliza algunas creencias, valores o actitudes en estos estudiantes, que sin duda influyen en su aprendizaje y en la aplicación futura de lo aprendido. La idoneidad afectiva analiza si se ha conseguido el interés y motivación del alumnado a lo largo del proceso de estudio, tanto por el tema en sí mismo, como por realizar las actividades propuestas.

- Idoneidad interaccional: Grado en que la organización de la enseñanza permite identificar conflictos y dificultades en el aprendizaje y resolverlos durante el proceso de instrucción. También, de acuerdo a principios constructivistas, se analiza la existencia de momentos en el proceso de estudio en que se apoye la autonomía y responsabilidad del estudiante.

- Idoneidad mediacional: Disponibilidad y adecuación de los recursos necesarios para el desarrollo del proceso de enseñanza-aprendizaje. Estos recursos pueden incluir la tecnología, materiales manipulativos, textos, tiempo dedicado al estudio, etc.

- Idoneidad ecológica: Se estudia si el proceso de estudio llevado a cabo es adecuado con respecto a los currículos oficiales y el proyecto educativo. También la forma en que se relaciona con otros temas matemáticos o extra matemáticos y si permite introducir innovaciones educativas.

Godino (2013) desarrolla con más detalle estos distintos componentes de la idoneidad didáctica y propone una guía de indicadores como instrumento de evaluación y formación de los profesores. Para ello se pediría a los profesores que analizasen algunos de los componentes de la idoneidad didáctica de un proceso de estudio de un contenido matemático observado en un aula o bien previamente experimentado por ellos mismos. De acuerdo a Godino, el análisis de la idoneidad epistémica por parte de los profesores permite al formador o al investigador valorar su 
conocimiento del contenido; su análisis de las idoneidades cognitiva y afectiva sirve para evaluar su conocimiento del contenido y el estudiante; el de la idoneidad interaccional y mediacional y ecológica, su conocimiento del currículo. Además, como consecuencia de esta actividad de análisis, se desarrolla el componente correspondiente del conocimiento del profesor.

En este trabajo nos proponemos evaluar el conocimiento del contenido y los estudiantes en una muestra de futuros profesores. Para ello, siguiendo la propuesta de Godino (2013) les pedimos valorar la idoneidad cognitiva y afectiva de un proceso de estudio que previamente han experimentado ellos mismos. En lo que sigue se expone el método y resultados obtenidos.

\section{Método}

La muestra estuvo formada por 108 futuros profesores de Educación Primaria de la Universidad de Granada, España, distribuidos en 3 grupos de clase (30 - 40 sujetos por grupo). Los datos se tomaron a partir de los informes individuales escritos que estos futuros profesores realizaron, como parte de una actividad práctica en una asignatura de didáctica de la matemática.

La actividad se llevó a cabo en dos sesiones de dos horas de duración cada una y separadas por un intervalo de una semana. En la primera sesión los participantes trabajaron individualmente para resolver el proyecto estadístico propuesto en el proceso de estudio. En la segunda, los participantes analizan la idoneidad afectiva y cognitiva del proceso de estudio en el que trabajaron en la primera sesión, utilizando una guía de análisis proporcionada por el formador (tomada de Godino, 2009). Los protocolos escritos producidos por los participantes individualmente en la segunda sesión son los que se analizan en este trabajo. A continuación se describe con más detalle el método seguido.

\subsection{Trabajo en la Sesión 1: Proceso de estudio de la estadística a través de un proyecto}

Como se ha indicado, durante la primera sesión los participantes resolvieron un proyecto de análisis de datos, en que trabajaron los contenidos elementales de estadística recogidos en el currículo de Educación Primaria: aleatoriedad, probabilidad simple, datos, recogida y representación gráfica, media, mediana, moda y rango. También se ejercitaron en la comparación de distribuciones utilizando las medidas de tendencia central y dispersión citadas. La sesión comenzó por una pregunta de investigación planteada por el formador, que motiva la recogida y análisis de datos tomados en la clase por los mismos sujetos participantes. Estos debían responder la pregunta basándose en los resultados del análisis de los datos y producir un informe escrito con sus conclusiones. La secuencia de actividades fue la siguiente:

- Presentación del proyecto y realización del experimento: El formador de profesores propuso a los estudiantes realizar un experimento para estudiar si el conjunto de estudiantes de la clase (los participantes) tenían o no buenas intuiciones sobre el azar. El experimento constó de dos partes: En la primera parte, cada participante tuvo que inventar y escribir una secuencia de 20 lanzamientos de una moneda sin realmente lanzar dicha moneda, de tal modo que otra persona pudiera pensar que se trataba de una secuencia aleatoria 
(secuencia simulada). En la segunda parte (secuencia real), los participantes anotaron los resultados de lanzar 20 veces una moneda.

- Recogida de datos: Finalizado el experimento, el formador de profesores inició un debate pidiendo a los participantes sugerencias para comparar las secuencias simuladas y reales generadas en el experimento en el total del grupo. Se acordó comparar las siguientes variables estadísticas: número de caras, número de rachas y longitud de la racha mayor en las secuencias real y simulada. Cada estudiante anotó los valores de estas seis variables en su propio experimento en una hoja de registro proporcionada por el profesor.

- Análisis de los datos: El formador proporcionó a cada participante una copia de la hoja de registro con los datos obtenidos por el conjunto de la clase para las variables descritas en el punto anterior y les pidió analizarlas como tarea para la siguiente semana. Los futuros profesores tuvieron que realizar un informe escrito con los resultados del análisis de los tres pares de variables estadísticas (número de caras, número de rachas y longitud de la racha más larga en las secuencias real y simulada de cada estudiante). Tuvieron libertad para el análisis de los datos. La mayoría realizaron tablas de frecuencia y diversos tipos de gráfico, como diagramas de barras o de líneas; generalmente, también se calculó la media, moda y rango de cada variable. Debían finalizar el informe con una conclusión sobre la intuición de la aleatoriedad en el conjunto de estudiantes.

- Conclusiones: La conclusión que se dedujo del análisis de los datos fue que los estudiantes tuvieron muy buena intuición sobre el valor esperado en el número de caras, pues las secuencias simuladas por ellos tuvieron un número medio de caras cercano al valor teórico 10. En cambio, su percepción de la variabilidad o del número de rachas fue mucho peor, ya que las secuencias eran poco variables y la longitud de las rachas muy cortas. Tanto el análisis como las conclusiones fueron presentadas y discutidas en la clase por los estudiantes hasta llegar a un consenso.

\subsection{Trabajo en la Sesión 2. Análisis de la idoneidad del proceso de estudio}

En la segunda sesión, los participantes tuvieron que valorar la experiencia de enseñanza que ellos mismos vivieron durante el desarrollo del proyecto. Para ello se dio a los futuros profesores una pauta de análisis de la idoneidad didáctica (Godino, 2009, 2013), en la cual se sugieren una serie de descriptores para analizar y concluir sobre la idoneidad global del proceso. El concepto de idoneidad didáctica y el uso de la pauta habían sido estudiados por los participantes, como parte de la asignatura, usando ejemplos de aplicación de los diferentes descriptores a otros temas. Cada futuro profesor trabajó individualmente, entregando al finalizar la actividad un informe escrito con el análisis realizado y sus conclusiones sobre la idoneidad didáctica de la experiencia.

En este trabajo analizamos la parte de los informes que se refiere a la valoración de las idoneidades cognitiva y afectiva (ver componentes y descriptores en las Tablas 1 y 2). Los participantes basaron su análisis en su experiencia personal al seguir el proceso de estudio de estadística consistente en la resolución del proyecto durante la Sesión 1 y a lo largo de la semana. 
Según Godino (2013), el análisis de estas dos componentes de la idoneidad didáctica por parte de profesores, les permite profundizar su conocimiento del contenido y de los estudiantes (en la terminología de Ball, y colaboradores), ya que han de reflexionar sobre los conocimientos previos de los estudiantes a los que va dirigida la enseñanza, así como las emociones, intereses y actitud de los mismos en relación al contenido particular, en este caso con respecto a la estadística elemental.

Tabla 1. Pauta de análisis de la idoneidad cognitiva de procesos de enseñanza y aprendizaje

\begin{tabular}{ll}
\hline \multicolumn{1}{c}{ Componentes } & \multicolumn{1}{c}{ Descriptores } \\
\hline Conocimientos previos & $\begin{array}{l}\text { CP1. Los estudiantes tienen los conocimientos previos } \\
\text { necesarios para el estudio del tema (se han estudiado } \\
\text { anteriormente o el profesor planifica su estudio). } \\
\text { CP2. Los contenidos pretendidos se pueden alcanzar (tienen } \\
\text { una dificultad manejable para los estudiantes). }\end{array}$ \\
$\begin{array}{l}\text { Adaptaciones curriculares a } \\
\text { las diferencias individuales }\end{array}$ & $\begin{array}{l}\text { AC1. Se incluyen actividades de ampliación y de refuerzo para } \\
\text { tener cuenta la atención a la diversidad de estudiantes. }\end{array}$ \\
& $\begin{array}{l}\text { AP1. Los resultados de la evaluación indican que los } \\
\text { estudiantes logran la apropiación de los conocimientos / } \\
\text { competencias pretendidos. }\end{array}$ \\
\hline
\end{tabular}

Tabla 2. Pauta de análisis de la idoneidad afectiva de procesos de enseñanza y aprendizaje

\begin{tabular}{ll}
\hline \multicolumn{1}{c}{ Componentes } & \multicolumn{1}{c}{ Descriptores } \\
\hline Intereses y & I1. Las tareas tienen interés para los estudiantes. \\
& $\begin{array}{l}\text { I2. Se proponen actividades que permitan valorar la utilidad de las } \\
\text { matemáticas en la vida cotidiana y profesional. }\end{array}$ \\
Actitudes & $\begin{array}{l}\text { ACT1. Se promueve la participación en las actividades, la perseverancia, } \\
\text { responsabilidad. }\end{array}$ \\
& $\begin{array}{l}\text { ACT2. Se favorece la argumentación en situaciones de igualdad; el } \\
\text { argumento se valora en sí mismo y no por quién lo dice. }\end{array}$ \\
Emociones & $\begin{array}{l}\text { E1. Se promueve la autoestima, evitando el rechazo, fobia o miedo a las } \\
\text { matemáticas. }\end{array}$ \\
& E2. Se resaltan las cualidades de estética y precisión de las matemáticas. \\
\hline
\end{tabular}

\subsection{Análisis de los datos}

A partir de los informes entregados por los futuros profesores se analizaron los párrafos que hicieron referencia a cada uno de los descriptores y componentes de las idoneidades cognitiva y afectiva (Tablas 1 y 2). A cada uno de estos párrafos se asignó un código que indica el nivel de análisis del futuro profesor de este descriptor. Se consideraron cuatro niveles:

Nivel 0: No se hace referencia al descriptor. Se deja la respuesta en blanco, no habiendo comprendido el descriptor o no siendo capaz de aplicarlo en la situación propuesta. 
Nivel 1: El futuro profesor se limita a copiar literalmente o casi literalmente la descripción del descriptor como aparece en la pauta, sin vincularlo con el proceso didáctico analizado.

Nivel 2: El participante aplica y hace referencia al descriptor, pero la aplicación es muy vaga y no refleja específicamente la situación particular de enseñanza que vivieron. Así, en el siguiente ejemplo, la futura profesora habla en general, sin especificar cuáles son los conocimientos aplicados en el proyecto que pueden resultar difíciles a los estudiantes

Los contenidos pretendidos se pueden alcanzar pero no en su totalidad ya que algunos de ellos son muy complejos (EA, descriptor CP2).

En otro ejemplo, JM aplica de manera incompleta el descriptor, pues se centra únicamente en el lanzamiento de la moneda para obtener datos, aunque en el desarrollo del proyecto hay muchos momentos en los que se promueven debates y la participación del alumnado:

Los estudiantes participan en el lanzamiento real de la moneda al aire (JM, descriptor ACT1).

Nivel 3: El participante hace referencia y aplica el descriptor relacionando los contenidos matemáticos trabajados para resolver el proyecto a lo largo de la Sesión 1, como el siguiente ejemplo:

Creo que para realizar la práctica, el estudiante debe tener unos conocimientos previos. Un ejemplo claro es la elaboración de gráficos estadísticos y el concepto de posición central y la dispersión. El cálculo de la media, mediana, moda y rango. Los conocimientos matemáticos necesarios para la realización de la práctica son de matemáticas de tercer ciclo de Educación Primaria (EA; descriptores CP1 y CP2).

En otro ejemplo, la futura profesora resalta la importancia de manejar contenidos estadísticos, como los gráficos, para la vida real:

Podemos valorar la utilidad que tienen las matemáticas, en cuanto a este tema, para la vida cotidiana y profesional, porque es útil saber manejar datos y gráficos (DB, descriptor I2).

\section{Análisis cualitativo de respuestas}

Una vez descritos los distintos niveles de aplicación de los descriptores, mostramos los resultados obtenidos. En primer lugar presentamos un análisis cualitativo en que se muestran ejemplos de respuesta y en segundo una síntesis cuantitativa de los resultados. Analizamos por separado las valoraciones realizadas de la idoneidad cognitiva y de la idoneidad afectiva.

\subsection{Valoración de la idoneidad cognitiva}

Sobre este componente de la idoneidad didáctica se entregó a los estudiantes los descriptores que aparecen en la Tabla 1, pidiéndoles que los valoraran en la situación analizada. El análisis de la idoneidad cognitiva del proyecto requiere de los futuros profesores valorar los conocimientos previos de los estudiantes a los que va dirigido el proyecto, la dificultad potencial de los nuevos conocimientos que se trabajan en el proyecto, la existencia de actividades de ampliación y refuerzo para atender a la 
diversidad de estudiantes y la evaluación del aprendizaje construidos por los estudiantes durante un proceso de estudio (Godino, 2013).

La mayoría de los participantes aplicaron los descriptores de la idoneidad cognitiva teniendo en cuenta su experiencia personal durante la Sesión 1, es decir, valoraron sus conocimientos previos y si el proyecto era adecuado para ellos como futuros profesores de Educación Primaria. En algunos casos, los participantes analizaron el proyecto considerando su aplicación en un aula de primaria, valorando si se adecuaba a las características cognitivas de estudiantes de Educación Primaria. Ambos casos son válidos para el objetivo de la práctica que era la adquisición de competencias para evaluar la idoneidad del proceso y no hubo diferencias relevantes en el nivel de aplicación de los descriptores entre las dos formas de valorar el proyecto.

Los componentes de la idoneidad cognitiva y sus descriptores se analizan a continuación, junto con algunos ejemplos de respuestas en que los participantes los aplican a diferentes niveles.

\section{Conocimientos previos y pretendidos}

CP1. Los estudiantes tienen los conocimientos previos necesarios para el estudio del tema (bien se han estudiado anteriormente o el profesor planifica su estudio). Al analizar este descriptor se espera que el futuro profesor reconozca que, para trabajar el proyecto planteado en la Sesión 1, sólo se requiere conocer las tablas y gráficos estadísticos elementales o bien incluso simplemente saber calcular la moda, media y rango. Todos estos conocimientos los poseen los estudiantes desde la escuela primaria, pues se incluyen en los decretos curriculares (MEC, 2006, MECD, 2014), Por consiguiente, en los últimos años de Educación Primaria, Educación Secundaria o en la formación de maestros se podría trabajar con este proyecto, realizando las adaptaciones oportunas dependiendo del nivel al que estuviese dirigido. Un ejemplo donde se aplica el descriptor a nivel 3 es el siguiente; pues CC indica la importancia de considerar si el estudiante tiene o no los conocimientos previos necesarios para el desarrollo de la tarea, indicando que el profesor trata de valorarlos en la primera sesión.

Han de tener en cuenta los conocimientos de conteo, y de las operaciones aritméticas elementales, que dependerán de cada estudiante. De todas formas, el tema de aleatoriedad será de las primeras veces que lo tratan y hará falta tratarlo con delicadeza y explicando todos los conceptos nuevos. En la primera actividad el profesor evalúa los conocimientos previos de los estudiantes, ya que formula preguntas sobre el tema que a continuación se van a abordar (CC, descriptor CP1).

CP2. Los contenidos pretendidos se pueden alcanzar (tienen una dificultad manejable para los estudiantes). Respecto a este descriptor, se espera que el futuro profesor señale que es posible alcanzar los contenidos pretendidos, ya que el proyecto es adecuado para estudiantes de últimos cursos de Educación Primaria, Educación Secundaria o formación de maestros. Más concretamente, en el desarrollo del proyecto se trató de trabajar los siguientes contenidos: Tratamiento de la información, aleatoriedad, experimentos y sucesos aleatorios, frecuencias, tablas, gráficos, medidas de posición central y dispersión.

En el siguiente ejemplo, EA aplica el descriptor a nivel 2 pues no hace referencia a contenidos específicos o a un nivel educativo dado. Su argumento puede ser válido si 
se refiere a estudiantes de Educación Primaria pero los contenidos citados no deberían ser un problema, cuando se trata de futuros profesores.

Los contenidos pretendidos se pueden alcanzar pero no en su totalidad ya que algunos de ellos son muy complejos para poder ser alcanzados por los alumnos (EA, descriptor $\mathrm{CP} 2$ ).

En este otro ejemplo, el estudiante JA aplica a nivel 3, estudiando conjuntamente los descriptores CP1 y CP2. Consideramos que su explicación es bastante completa, ya que hace referencia explícita a conocimientos previos que deberían poseer los estudiantes para la correcta realización del proyecto y destaca que dichos conocimientos previos los deberían poseer estudiantes de tercer ciclo de Educación Primaria. Además basándose en la información proveniente de los documentos curriculares para la Educación Primaria (MEC, 2006; MECD, 2014), resalta que los contendidos pretendidos que se explicitan en la unidad didáctica pueden ser alcanzados en últimos cursos de Educación Primaria, teniendo estos, una "dificultad manejable".

Creo que para realizar la práctica, el estudiante, debe tener los conocimientos previos que se necesitan para trabajar en el proyecto, en el cuál se deben utilizar fórmulas y definiciones muy concretas, conocidas para él. Un ejemplo claro es la elaboración de gráficos estadísticos y el concepto de posición central y dispersión. Otro ejemplo el cálculo de la media, mediana, moda y rango. Todos estos conocimientos, necesarios para la realización de la actividad, son de matemáticas de tercer ciclo de Educación Primaria. Los contenidos pretendidos deben de ser fácilmente accesibles para estudiantes del tercer ciclo de Educación Primaria, puesto que se incluyen en el Decreto de Enseñanzas Mínimas en Educación Primaria. Se puede decir que el proyecto tiene una dificultad manejable (JA, descriptores CP1 y CP2).

\section{Atención a la diversidad}

AC1. Se incluyen actividades de ampliación y de refuerzo para tener en cuenta la atención a la diversidad de estudiantes. Se espera que el futuro profesor, en su análisis, identifique las actividades de ampliación optativas que se incluyeron en el proyecto trabajado en la Sesión 1. En este sentido algunos participantes opinaron que las actividades eran insuficientes; otros consideraron que la actividad propuesta es suficiente e idónea, mostrando esto la diversidad de opiniones que pueden aparecer ante una misma actividad de formación de profesores.

Un ejemplo de respuestas en que el futuro profesor aplica este descriptor a nivel 2 se muestra a continuación. JMG considera que, una vez realizado el experimento y recogidos los datos (sobre las secuencias reales y simuladas), la realización de gráficos serían actividades de ampliación. Este participante no comprende que los cálculos y gráficos realizados con estos datos son necesarios para comparar las distribuciones de distintas variables estadísticas, es decir, no se trata de actividades de ampliación, sino parte del proyecto.

Las actividades de ampliación han sido la realización de gráficos con los valores de las secuencias (JMG, descriptor AC1).

Por el contrario, MEV, aplica el descriptor en un sentido más adecuado (nivel 3), observando la existencia de actividades de ampliación y refuerzo a lo largo de la unidad didáctica.

En este proyecto si se incluyen actividades de ampliación y refuerzo; para ello se ha dejado un apartado exclusivamente dedicado a actividades de ampliación y refuerzo, 
como, por ejemplo, realizar nuevos gráficos o analizar otras variables (MEV, descriptor AC1).

\section{Evaluación del aprendizaje}

AP1. Los resultados de la evaluación indican que los estudiantes logran la apropiación de los conocimientos / competencias pretendidos. En la primera parte de la segunda sesión se dedicó media hora a discutir las soluciones aportadas al proyecto por parte de los participantes en el informe escrito realizado en la primera sesión. Además, el formador de profesores dispuso de dichos informes escritos para evaluar individualmente a cada participante. Por tanto, se esperaba que los futuros profesores, al analizar este descriptor reconociesen que se hizo una evaluación y también personalmente valorasen su propio aprendizaje, como consecuencia de la realización del proyecto.

Consideramos que este descriptor se aplica a nivel 2 cuando el futuro profesor no se centra en los aprendizajes o en las tareas de evaluación, como ocurre en el siguiente ejemplo. En él, el participante sólo tiene en cuenta el desarrollo de la primera sesión, pero no los informes escritos que permitirán al formador evaluar el aprendizaje de cada participante; tampoco da su opinión sobre si este aprendizaje se alcanza o no:

No se hace una evaluación como tal, aunque el profesor va anotando datos de los estudiantes durante la secuencia de actividades y los usará para dar unas calificaciones de cada uno según su actitud y resolución ante la propuesta (JA, descriptor AP1).

A nivel 3 el estudiante aplica el descriptor, destacando las correcciones llevadas a cabo por el formador de profesores durante el desarrollo de la práctica, comentando los métodos de evaluación propuestos, o bien realizando sugerencias de cambio de dichos métodos con el fin de fomentar un mejor aprendizaje por parte de los estudiantes. Por ejemplo, la alumna MEF, comenta los instrumentos de evaluación propuestos en la unidad didáctica, sugiriendo una mayor observación individualizada de los estudiantes.

En la práctica se han utilizado varios instrumentos de evaluación. Creo que los ejercicios realizados son valiosos, pero yo lo ampliaría con varios ejercicios más, o si es posible, observaría a cada uno de los estudiantes, independientemente, para completar la información que tenemos sobre su comprensión del tema (MEF, descriptor AP1).

\subsection{Valoración de la idoneidad afectiva}

Según Godino (2013) la idoneidad afectiva está relacionada tanto con factores que dependen de la institución, como con otros que dependen básicamente del alumnado y de su historia escolar previa. Al analizarla, los futuros profesores han de tener en cuenta el grado de implicación, interés y motivación que ellos mismos tuvieron con relación al desarrollo del proyecto estadístico. Para valorarla se propusieron a los futuros profesores analizar descriptores relacionados con el interés de la actividad para los estudiantes, las actitudes positivas que se promueven mediante el trabajo con el proyecto y las posibles emociones que suscita. A continuación se analizan dichos descriptores, junto con algunos ejemplos de respuestas de los futuros profesores a los mismos. 


\section{Interés para el estudiante}

I1. Las tareas tienen interés para los estudiantes. Se esperaba que los futuros profesores se implicasen en la dinámica de la realización del proyecto y se sintiesen interesados en esta forma de trabajar la estadística. Se esperaba también que los futuros profesores apreciasen que los proyectos aumentan la motivación de los estudiantes (Holmes, 1997), puesto que los datos surgen de un problema relevante para ellos, les pueden dotar de significado y los resultados estadísticos tienen que ser interpretados y relacionados con la realidad. Por otro lado, el tema del proyecto, y la constatación de sus intuiciones incorrectas respecto al azar, podría resultarles de interés.

En el ejemplo siguiente, se aplica el descriptor a nivel 2 pues PC se centra en aspectos anecdóticos, ya que relaciona la estadística con los intereses de los estudiantes y la vida cotidiana, pero lo hace en general y no en particular para el proyecto que realizaron. Por ello esta respuesta no puede ser considerada dentro del nivel siguiente, ya que es imprecisa.

Dado que la estadística está relacionada con hechos de la vida cotidiana provoca un mayor grado de interés por parte del estudiante (PC, descriptor I1).

I2. Se proponen situaciones que permitan valorar la utilidad de las matemáticas en la vida cotidiana y profesional. En relación con este descriptor los participantes podrían valorar la utilidad de la estadística a la hora de resolver un proyecto, que puede ser considerado como una pequeña investigación (McGilliwray \& Pereira-Mendoza, 2011). Además se esperaba que constatasen que el análisis de datos y el trabajo con representaciones gráficas puede ser de gran utilidad para formar ciudadanos cultos estadísticamente (Ridgway, Nicholson \& McCusker, 2008). La alumna DB aplica el descriptor a nivel 3, pues resalta que en determinados trabajos puede ser de gran utilidad los conocimientos trabajados con el proyecto. En particular, destaca el trabajo con gráficos estadísticos como útil para la vida real.

Podemos valorar la utilidad que tienen las matemáticas, en cuanto a este tema, para la vida cotidiana y profesional, ya que en un futuro para diversos trabajos necesitarán lo que hemos trabajado en el proyecto, como saber calcular la media y mediana. Los niños en el colegio, al realizar esa actividad se darán cuenta de la importancia que tiene la matemática en la vida real, porque es útil saber manejar datos y gráficos (DB, descriptor I2).

\section{Actitudes}

ACT1. Se promueve la participación en las actividades, la perseverancia, responsabilidad, etc. Se trata de evaluar si el proyecto propuesto facilita la participación en diversas actividades a lo largo de su realización. Se espera que los futuros profesores comenten la forma en que se promovió la participación y la responsabilidad en distintas fases del proyecto, como, por ejemplo, la realización del experimento, el cual participaron todos los estudiantes de la clase. Además, después de la recogida de datos, cada uno tuvo que realizar un informe individual para responder a una pregunta, por lo que tuvieron que ser responsables en la realización de la tarea, pues se les dio una semana para que entregasen sus resultados.

En el siguiente ejemplo, EA, aplica el descriptor ACT1 a nivel 2, pues lo hace de una manera muy imprecisa, ya que, aunque es verdad que los estudiantes participaron en el lanzamiento real de la moneda, no hace referencia en ningún momento a si el proyecto fomenta o no la participación de los estudiantes. 
Los estudiantes participan en el lanzamiento real de la moneda al aire (EA, descriptor ACT1).

ACT2. Se favorece la argumentación en situaciones de igualdad; el argumento se valora en sí mismo y no por quién lo dice. En la implementación del proyecto se hicieron varios debates, en los cuales todos pudieron dar su opinión, respetándose las opiniones y argumentos que se pusieron en juego. Por tanto la actividad tiene fases en las que claramente los estudiantes pueden argumentar sus ideas delante de todos sus compañeros. Un ejemplo de aplicación a nivel 3 es el siguiente:

Aquí se ven claramente que se promueve la participación en las actividades de todos los compañeros al hacer preguntas del tipo: ¿Cuántas veces hay que lanzarla? ¿Es normal que me salgan 4 caras seguidas? ... se anima a los estudiantes a estar atentos de los compañeros. Vemos esta actitud participativa en la página 5 en el ejercicio 4, al hablar de comparar resultados con los de tus compañeros, analizar los datos, ayudar al profesor a recoger datos. Se favorece, como no, la responsabilidad al hacer que el estudiante esté pendiente de su moneda, de que haga los lanzamientos pedidos, los anote correctamente. También al hacerle reflexionar sobre las soluciones obtenidas, se fuerza a que el estudiante se haga preguntas sobre ellas, se responsabilice de lo que le ha salido y lo comprenda para poder explicarlo (Estudiante JM, descriptor ACP1).

\section{Emociones}

E1. Se promueve la autoestima, evitando el rechazo, fobia o miedo a las matemáticas. Los futuros profesores han de valorar si el proyecto anima a los estudiantes a trabajar y reconozcan que el trabajo con proyectos estadísticos y la realización de experimentos aleatorios en el aula es un vehículo para que superen su posible miedo a enfrentarse a problemas de estadística (Starkings, 1997).

En el siguiente ejemplo AG aplica este descriptor a nivel 3, pues destaca que el hecho de que los estudiantes realicen un proyecto para responder a una pregunta de investigación y recojan sus propios datos, resulta de interés para los estudiantes. Por ello despierta su autoestima, evitando el rechazo a trabajar temas de estadística, viendo la utilidad de esta. Estas posibilidades del trabajo con proyectos estadísticos ya han sido analizadas en Díaz, Arteaga y Batanero (2007).

Se promueve la autoestima, evitando el rechazo, fobia o miedo a las matemáticas, ya que las actividades se hacen a través de la práctica del lanzamiento de la moneda, y de este modo los estudiantes ven lo que ocurre, es algo real, no abstracto y les llama la atención (AG, descriptor E1).

E2. Se resaltan las cualidades de estética y precisión de las matemáticas. Durante la realización de la actividad, los futuros profesores realizaron un informe escrito, en el que realizaron gráficos para resolver el problema planteado. La mayoría de ellos se esforzaron en la claridad y presentación estética de dichos gráficos, utilizando colores y otros elementos visuales para diferenciar las variables y resaltar sus características. Esperamos que los futuros profesores valoren que, por medio de esta actividad, no sólo se resaltan las cualidades estéticas de los gráficos, sino en general de las matemáticas. Respecto al tema de la precisión, aunque la probabilidad es la parte de la matemática que modeliza la incertidumbre, esperamos que también los estudiantes lo reconozcan, así como que las cualidades de precisión y aproximación se complementan en matemáticas. En el siguiente ejemplo, sin embargo, la alumna TA aplica el descriptor a nivel 2, llegando a la conclusión que la actividad no resalta ni la estética ni la precisión de las matemáticas y 
además contrapone probabilidad con precisión.

No se resaltan cualidades de estética y precisión ya que la probabilidad no es precisa ni exacta y existen distintas combinaciones (TA, descriptor E2).

\section{Síntesis cuantitativa y discusión de resultados}

Una vez presentado el análisis cualitativo de las respuestas, en esta sección estudiaremos dos tipos de indicadores cuantitativos del conocimiento mostrado por los futuros profesores: Por un lado presentaremos los porcentajes de participantes que valoran cada uno de los descriptores presentados en los diferentes niveles descrito. Por otro, se presenta un gráfico comparado del nivel medio del grupo en cada distractor. El análisis se completa con una discusión de los resultados.

\subsection{Síntesis de los resultados de la evaluación de la idoneidad cognitiva}

En la Tabla 3 se muestra la frecuencia de aplicación de los niveles en cada uno de los descriptores de la idoneidad cognitiva. El descriptor CP1 (conocimientos previos necesarios) fue el más fácil de aplicar, ya que un $81,48 \%$ de los futuros profesores lo aplican al menos a nivel 2. Es decir, prácticamente todo el grupo fue capaz de reconocer que los conocimientos estadísticos requeridos para trabajar con el proyecto se habían adquirido previamente. Asimismo muchos estudiantes pudieron reconocer que estos contenidos (gráficos estadísticos elementales, media, rango) se incluyen en las orientaciones curriculares para la Educación Primaria (MEC, 2006; MECD, 2014).

Además, en una proporción alta llegan al nivel tres de aplicación de este descriptor; por tanto identificaron los conocimientos matemáticos concretos contenidos en el proyecto. Este resultado es bastante mejor que el obtenido en investigaciones previas, como la de Chick y Pierce (2008) quienes indican que los profesores de su estudio no fueron capaces de identificar los contenidos matemáticos relevantes en los proyectos estadísticos trabajados con sus estudiantes.

Un número menor de participantes analizan los descriptores CP2; si los contenidos pretendidos parecen alcanzables y AP1; si los resultados de la evaluación indica que se logra el aprendizaje. Aunque las soluciones escritas al proyecto permiten evaluar los conocimientos alcanzados por los estudiantes, al no haber una evaluación previa de los conocimientos estadísticos, los futuros profesores no fueron capaces de determinar si hubo aprendizaje en el grupo.

Tabla 3. Frecuencia (porcentaje) de nivel de aplicación de descriptores de la idoneidad cognitiva

\begin{tabular}{ccccc}
\hline Aplicación del descriptor & CP1 & CP2 & AC1 & AP1 \\
\hline Nivel 0 & $6(5,56)$ & $54(50,00)$ & $30(27,78)$ & $44(40,74)$ \\
Nivel 1 & $14(12,96)$ & $11(10,19)$ & $22(20,37)$ & $10(9,26)$ \\
Nivel 2 & $44(40,74)$ & $19(17,59)$ & $21(19,44)$ & $27(25,00)$ \\
Nivel 3 & $44(40,74)$ & $24(22,22)$ & $35(32,41)$ & $27(25,00)$ \\
\hline
\end{tabular}

En del descriptor AC1 que trataba valorar las adaptaciones a la diversidad, sube el porcentaje de participantes que lo aplica a nivel 3 , es decir, que valoraron adecuadamente las actividades de ampliación y refuerzo de la unidad didáctica. Estos 
estudiantes también apreciaron las posibilidades de los proyectos estadísticos para que cada alumno pueda trabajar en forma diferenciada, dependiendo de su conocimiento previo, que ha sido señalada por autores como Starkings (1997).

Para proporcionar otro resumen cuantitativo de la capacidad de análisis de la idoneidad cognitiva presentamos en la Figura 1 las puntuaciones medias del grupo en cada descriptor. Al estudiar dichas puntuaciones medias, destaca el nivel de aplicación del descriptor CP1 $(2,17)$, que fue el más fácil de aplicar. Ello fue debido a que los futuros profesores tuvieron más facilidad de reconocer los conocimientos previos necesarios al elaborar el proyecto, que el aprendizaje conseguido. La puntuación del resto de descriptores se sitúa entre 1 y 1,5, debido al alto porcentaje de estudiantes que no llegan a analizar dichos descriptores (CP2, AC1 y AP1). Pensamos que los problemas y dificultades que ellos mismos tuvieron en su resolución, Por ejemplo, los alumnos tuvieron errores en las escalas de los gráficos utilizados o en su lectura, similares a los descritos por Espinel (2007). Dichas dificultades incidieron en que no supiesen valorar adecuadamente el aprendizaje conseguido.

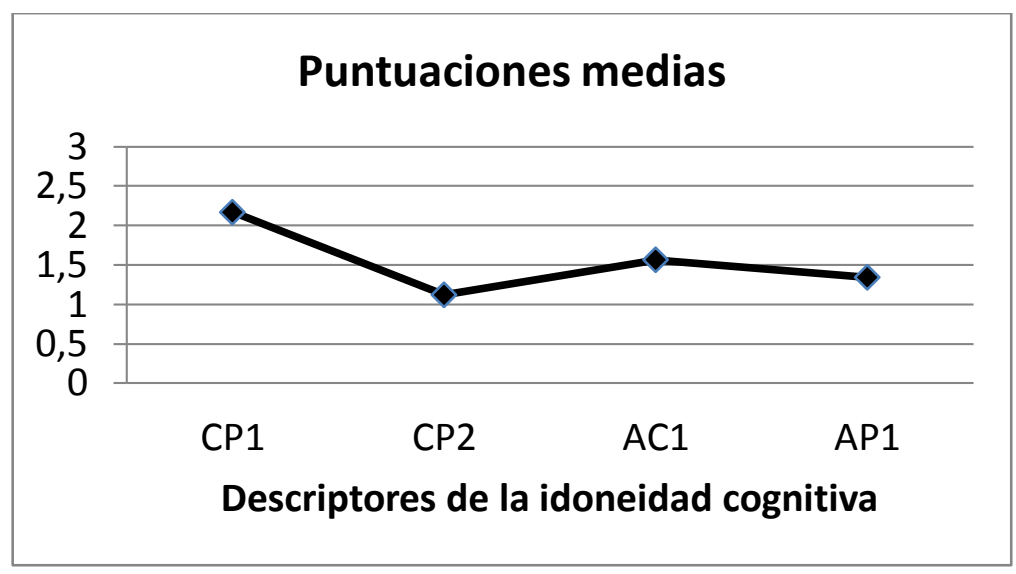

Figura 1. Puntuaciones medias en los descriptores de la idoneidad cognitiva

\subsection{Síntesis de los resultados de la evaluación de la idoneidad afectiva}

En la Tabla 4 mostramos el porcentaje de futuros profesores que aplica los descriptores de la idoneidad afectiva según los distintos niveles. El más sencillo de aplicar fue el descriptor I1, relacionado con el interés de los estudiantes, debido a que ellos mismos se interesaron mucho en el proyecto. Al trabajar con una metodología diferente de la habitual y al tratarse de unos datos recogidos por ellos mismos y relativos a sus propias intuiciones, los alumnos se sintieron en todo momento muy motivados para completar el proyecto y conocer los resultados del análisis de los datos. Hall (2011) sugiere la importancia del uso de datos reales en el trabajo con la estadística, pues los estudiantes aprenderán sobre la recogida de datos, la importancia de los datos faltantes o atípicos y de la validez y fiabilidad de los datos. Igualmente permiten trabajar temas interdisciplinares, como se hizo en el proyecto utilizado en esta investigación.

También se obtuvieron resultados relativamente buenos en la valoración del descriptor ACT1 (el proyecto contribuye a la responsabilidad y participación). Los estudiantes efectivamente asumieron la responsabilidad del análisis de datos, pues el profesor les dio libertad sobre la forma de llevarlo a cabo. Participaron activamente tanto en la recogida de datos como en su análisis, ideando sus propios gráficos y presentando 
un informe estadístico detallado de sus resultados. Los futuros profesores reconocieron estas dos características en sus respuestas de valoración de este descriptor.

El porcentaje de participantes que no aplica los descriptores crece mucho en los restantes descriptores, llegando al 58,33\% en el descriptor ACT2 relacionado con las actitudes y un $69,44 \%$ en el descriptor E2 relacionado con las emociones y las cualidades estéticas de las matemáticas. Los futuros profesores mostraron su poca formación en los aspectos afectivos de las matemáticas; siendo el tema de actitudes relativamente desconocido para ellos. Por otro lado, usualmente el aspecto estético de las matemáticas se asocia más al campo de la geometría que al de la estadística, lo que llevó a algunos estudiantes a no saber responder en este punto.

Tabla 4. Frecuencia (porcentaje) de nivel de aplicación de descriptores de la idoneidad afectiva

\begin{tabular}{ccccccc}
\hline Aplicación del descriptor & I1 & I2 & ACT1 & ACT2 & E1 & E2 \\
\hline Nivel 0 & $11(10,19)$ & $40(37,04)$ & $27(25,00)$ & $63(58,33)$ & $46(42,59)$ & $75(69,44)$ \\
Nivel 1 & $27(25,00)$ & $22(20,37)$ & $31(28,70)$ & $14(12,96)$ & $17(15,74)$ & $13(12,04)$ \\
Nivel 2 & $21(19,44)$ & $19(17,59)$ & $12(11,11)$ & $9(8,33)$ & $19(17,59)$ & $14(12,96)$ \\
Nivel 3 & $49(45,37)$ & $27(25,00)$ & $38(35,19)$ & $22(20,37)$ & $26(24,07)$ & $6(5,56)$ \\
\hline
\end{tabular}

Para resumir los resultados del análisis de la idoneidad afectiva, en la Figura 2 se muestran los valores medios obtenidos por los futuros profesores de nuestra muestra al aplicar los distintos descriptores de dicha idoneidad. Observamos una variedad en los niveles de aplicación, siendo el más fácil de aplicar el I1 (si el proyecto suscitaba el interés de los estudiantes), debido a que, como se ha dicho, el proyecto les interesó bastante. El más difícil fue el E2 (cualidades estéticas y de precisión de las matemáticas en el caso particular del proyecto). La aplicación de los descriptores de la idoneidad afectiva resultó difícil a los futuros profesores, siendo el nivel promedio de aplicación menor de 2 en todos los descriptores salvo en el primero. No obstante, tanto el descriptor I1 como el ACT1 tuvieron un considerable porcentaje de aplicación a nivel 3.

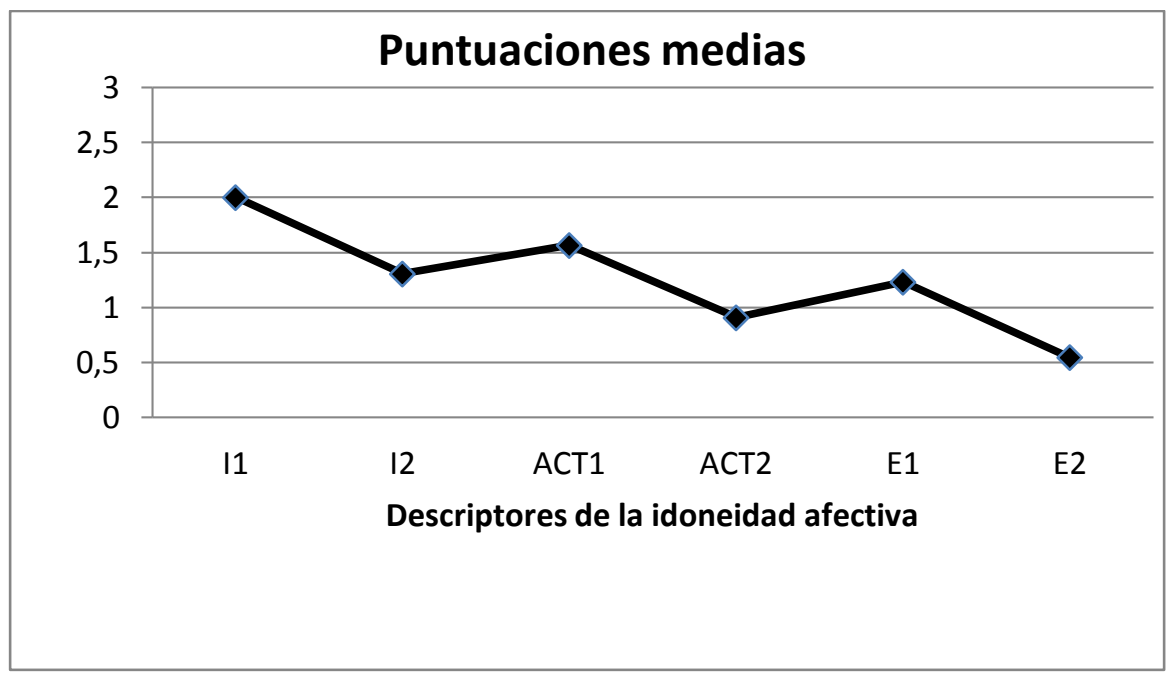

Figura 2. Puntuación media en los descriptores de la idoneidad afectiva 


\section{Conclusiones}

En un estudio previo (Arteaga, Batanero, Cañadas, et al., 2012) mostramos las dificultades que futuros profesores de Educación Primaria tenían al analizar la idoneidad epistémica de un determinado proceso de enseñanza y aprendizaje de la estadística. Indirectamente se mostró su escaso conocimiento especializado de la estadística, que es necesario para la planificación de las clases por parte del profesor. Los datos de este nuevo estudio indican que, aunque hay determinados descriptores que resultaron fáciles de aplicar, globalmente, los futuros profesores de nuestra muestra también tienen dificultades en evaluar las idoneidades cognitiva y afectiva. Indirectamente se observa que los participantes en este estudio muestran escaso conocimiento de la estadística y los estudiantes pues no son capaces de valorar los conocimientos previos, el aprendizaje o las actitudes de los mismos.

Estos resultados complementan otras investigaciones previas, que muestran que el conocimiento común de la estadística es pobre en los futuros profesores de Educación Primaria (Espinel, 2007; Arteaga y Batanero, 2010). Ello sin duda influye en el escaso conocimiento del contenido y el estudiante mostrado por los participantes en nuestra investigación, pues el conocimiento del contenido es un requisito esencial para alcanzar un conocimiento suficiente sobre el contenido y los estudiantes.

Como consecuencia de toda esta investigación, se hace evidente la necesidad de mejorar la formación de profesores de Educación Primaria, en lo que respecta a sus conocimientos para enseñar estadística. En particular se hace necesario mejorar su conocimiento sobre los estudiantes, es decir, cómo los estudiantes aprenden, las dificultades que tienen con un determinado contenido; así como cuáles son sus actitudes, intereses y emociones y el modo en que todos estos puntos se relacionan con el trabajo con la estadística. La importancia de los aspectos afectivos en el estudio de las matemáticas es destacada, entre otros por Estrada, Bazán y Aparicio (2013) y Pérez-Tyteca, Monje y Castro (2013). Sin embargo, en nuestro estudio fue visible el desconocimiento de los alumnos de las actitudes o de sus componentes; uno de los descriptores básicos del dominio afectivo de las matemáticas, según Gómez-Chacón (2000).

El reto de la preparación específica de los profesores para enseñar estadística fue reconocido en el estudio de ICMI e IASE resumido en Batanero, et al. (2011). Sus conclusiones señalan las carencias formativas, proponen diversas acciones para resolverlas e indican que sólo con una preparación suficiente será posible abordar con éxito la enseñanza de la estadística en la escuela.

Esperamos que dicho estudio, así como los resultados mostrados en este trabajo, ayuden a tomar conciencia de esta necesidad a todos los implicados en la formación de profesores: Escuelas de Formación del Profesorado, asociaciones de profesores y autoridades educativas. Creemos también que es necesario continuar con la investigación y reflexión didáctica para poder seguir construyendo la Educación Estadística y concretándola en cursos destinados a futuros profesores.

\section{Agradecimientos}

Proyecto EDU2013-41141-P (MEC), y grupo FQM126 (Junta de Andalucía). 


\section{Referencias bibliográficas}

Arteaga, P., \& Batanero, C. (2010). Evaluación de errores de futuros profesores en la construcción de gráficos estadísticos. En M. Moreno, A. Estrada, J. Carrillo, \& T. Sierra (Eds.), XII Simposio de las Sociedad Española de Investigación en Educación Matemática (pp. 211-221). Lleida: SEIEM.

Arteaga, P., Batanero, C., Contreras, J. M., \& Cañadas, G. R. (2012). Evaluación del conocimiento de la estadística y los estudiantes en futuros profesores. En A. Estepa, Á. Contreras, J. Deulofeu, C. Penalva, F. J. García. \& L. Ordóñez (Eds.), Investigación en Educación Matemática XVI (pp. 135-143). Jaén: Sociedad Española de Investigación en Educación Matemática.

Arteaga, P., Batanero, C., Cañadas, G. R., \& Gea, M. M. (2012). Evaluación del conocimiento especializado de la estadística en futuros profesores mediante el análisis de un proyecto estadístico. Educaçao Matemática Pesquisa, 14 (2), 279297.

Batanero, C., \& Díaz, C. (2010). Training teachers to teach statistics: what can we learn from research? Statistique et Enseignement, 1(1), 5-20. Disponible en: www.statistique-et-enseignement.fr/ojs/.

Batanero, C., Burrill, G., \& Reading, C. (2011). Teaching Statistics in School Mathematics. Challenges for Teaching and Teacher Education: A Joint ICMI/IASE Study. New York. Springer.

Ball, D. L., Thames, M. H., \& Phelps, G. (2005, Abril). Articulating domains of mathematical knowledge for teaching. Paper presented at the Annual Meeting of the American Educational Research Association. Montereal, QC, Canadá.

Burgess, T. (2006). A framework for examining teacher knowledge as used in action while teaching statistics. En A. Rossman, \& B. Chance (Eds.), Proceedings of the Seventh International Conference on Teaching Statistics. Salvador, Brazil: International Statistical Institute.

Chick, H. L. \& Pierce, R. U. (2008). Teaching statistics at the primary school level: beliefs, affordances, and pedagogical content knowledge. En C. Batanero, G. Burrill, C. Reading \& A. Rossman (Eds.), Joint ICMI/IASE Study: Teaching Statistics in School Mathematics. Challenges for Teaching and Teacher Education. Proceedings of the ICMI Study 18 and 2008 IASE Round Table Conference. Monterrey, Mexico: ICMI e IASE. Online: www.ugr.es/ icmi/iase_study/.

Common Core State Standards Initiative (CCSSI) (2010). Common Core State Standards for Mathematics. Washington, DC: National Governors Association for Best Practices and the Council of Chief State School Officers. Disponible en: www.corestandards.org/assets/CCSSI_Math Standards.pdf.

Dawson, A. J., Jaworski, B., \& Wood, T. (Eds.). (2013). Mathematics teacher education: Critical international perspectives. Dordrecht, The Netherlands; Routledge.

Díaz, C., Arteaga, P., \& Batanero, C. (2007). Contribución del trabajo con proyectos estadísticos a la adquisición de competencias básicas. En M. Molina, P. PérezTyteca, \& M. A. Fresno (Eds.), Jornadas de Investigación en el Aula de Matemáticas. Competencias matemáticas [CD- ROM]. Granada. Sociedad Thales y Departamento de Didáctica de las Matemáticas. 
Espinel, C. (2007). Construcción y razonamiento de gráficos estadísticos en la formación de profesores. Investigación en Educación Matemática, 11, 99-119.

Estrada, A., Bazán, J. \& Aparicio, A. (2013). Evaluación de las propiedades psicométricas de una escala de actitudes hacia la estadística en profesores. Avances de Investigación en Educación Matemática, 3, 5-23.

Even. R., \& Ball, D. (2009). The professional education and development of teachers of mathematics. The 15th ICMI Study. New York: Springer.

Franklin, C., Kader, G., Mewborn, D., Moreno, J., Peck, R., Perry, M, \& Scheaffer, R. (2007). Guidelines for assessment and instruction in statistics education (GAISE) report: A Pre-K-12 curriculum framework. Alexandria, VA: American Statistical Association. Disponible en: www.amstat.org/Education/gaise/.

Godino, J. D. (2009). Categorías de análisis de los conocimientos del profesor de matemáticas. Unión, 20, 13-31.

Godino, J. D. (2013). Indicadores de la idoneidad didáctica de procesos de enseñanza y aprendizaje de las matemáticas. Cuadernos de Investigación y Formación en Educación Matemática, 11, 111-132.

Godino, J. D., Batanero, C., Rivas, H., \& Arteaga, P. (2013). Componentes e indicadores de idoneidad de programas de formación de profesores en didáctica de las matemáticas. Revemat, 8(1), 46-74

Godino, J. D., Contreras, A., \& Font, V. (2006). Análisis de procesos de instrucción basado en el enfoque ontológico-semiótico de la cognición matemática. Recherches en Didactiques des Mathematiques, 26(1), 39-88.

Gómez-Chacón, I. M. (2000). Matemática emocional. Los afectos en el aprendizaje matemático. Narcea. Madrid

Hall, J. (2011). Engaging teachers and students with real data: Benefits and challenges. In C. Batanero, G. Burrill, \& C. Reading (Eds.), Teaching statistics in school mathematics. Challenges for teaching and teacher education. A joint ICMI/IASE Study (pp. 335-346). New York: Springer.

Hill, H. C., Ball, D. L., \& Schilling, S. G. (2008). Unpacking pedagogical content knowledge: Conceptualizing and measuring teachers' topic-specific knowledge of students. Journal for Research in Mathematics Education, 39, 372-400.

Hill, H., Schilling, S., \& Ball, D. L. (2004). Developing measures of teachers' mathematical knowledge for teaching. Elementary School Journal, 105(1), 11-30.

Hill, H. C., Sleep, L., Lewis, J. M., \& Ball, D. (2007). Assessing teachers' mathematical knowledge. En F. Lester (Ed.), Second handbook of research on mathematics teaching and learning (pp. 111-155). Greenwich, CT: Information Age Publishing, Inc. y NCTM.

Holmes, P. (1997). Assessing project work by external examiners. En I. Gal, \& J. B. Garfield (Eds.), The assesment challenge in statistics education (pp. 153-164). Voorburg: IOS Press.

LLinares S., \& Krainer K. (2006). Mathematics (student) teachers and teacher educators as learners. En A. Gutierrez, \& P. Boero (Eds), Handbook of Research on the Psychology of Mathematics Education (pp. 429 - 459). Rotherdam / Taipei: Sense Publishers. 
MacGillivray, H., \& Pereira Mendoza, L. (2011). Teaching statistical thinking through investigative projects. En C. Batanero, G. Burrill, \& C. Reading, (Eds.), Teaching statistics in school mathematics. Challenges for teaching and teacher education. A joint ICMI and IASE study (pp. 109-120). New York: Springer.

Ministerio de Educación y Ciencia, MEC (2006). Real Decreto 1513/2006, de 7 de diciembre, por el que se establecen las enseñanzas mínimas correspondientes a la Educación Primaria.

Ministerio de Educación, Cultura y Deporte, MECD (2014). Real Decreto 126/2014, de 28 de febrero, por el que se establece el currículo básico de la Educación Primaria. Madrid: Autor.

National Council of Teachers of Mathematics, NCTM (2000). Principles and standards for school mathematics. Reston, VA: Author.

Pérez-Tyteca, P., Monje, J. \& Castro, E. (2013). Afecto y matemáticas. Diseño de una entrevista para acceder a los sentimientos de alumnos adolescents. Avances de Investigación en Educación Matemática, 4, 65-82.

Pierce. R., \& Chick, J. (2011). Teachers' beliefs about statistics education. En C. Batanero, G. Burrill, \& C. Reading, (Eds.), Teaching statistics in school mathematics. Challenges for teaching and teacher education. A joint ICMI and IASE study (pp. 151-162). New York: Springer.

Pinto, J. (2010). Conocimiento didáctico del contenido sobre la representación de datos estadísticos: Estudios de casos con profesores de estadística en carreras de psicología y educación. Tesis Doctoral. Universidad de Salamanca.

Ridgway, J., Nicholson, J., \& McCusker, S. (2008). Mapping new statistical Literacies and Iliteracies. Trabajo presentado en el 11th International Congress on Mathematics Education, Monterrey, Mexico.

Starkings, S. (1997). Assessing students' projects. En I. Gal, \& J. B. Garfield (Eds.), The assesment challenge in statistics education (pp. 139-152). Voorburg: IOS Press.

Vygotski, L.S. (1934). El desarrollo de los procesos psicológicos superiores. Barcelona: Crítica-Grijalbo.

Wood, T. (Ed.) (2008). The international handbook of mathematics teacher education. Rotterdam: Sense Publishers.

\section{Referencia a los autores}

Pedro Arteaga, Universidad de Granada (España), parteaga@ugr.es

J. Miguel Contreras, Universidad de Granada (España), jmcontreras@ugr.es

Gustavo R. Cañadas, Universidad de Granada (España), grcanadas@ugr.es 


\title{
Knowledge of statistics and students in prospective school teachers: an exploratory study
}

\author{
Pedro Arteaga, Universidad de Granada (España) \\ J. Miguel Contreras, Universidad de Granada (España) \\ Gustavo R. Cañadas, Universidad de Granada (España)
}

The aim of this research was to analyze the knowledge of statistics and students of a sample of 108 prospective pre-service primary school teachers. Knowledge of statistics and students is one component in the mathematical knowledge for teaching model (Hill, Ball, \& Schilling, 2008) and describes used by the teacher to teach a particular mathematical content to particular students as learners. This knowledge involves understanding students' mathematical work and thinking, their preconception and prior knowledge that students bring to the classroom, as well as their interests and attitudes. In this work we reinterpret this component in the sense suggested by Godino $(2009 ; 2013)$ as the union of the cognitive and affective dimensions of his model of didactic- mathematics knowledge for teaching.

Data were collected as a part of a practical activity in two sessions. In the first session the prospective teachers worked with a statistical project where they had to collect and analyze data using elementary statistics. In the second session, the participants were asked to analyze the different components and descriptors of the project cognitive and affective suitability (Godino, 2009, 2011).

The qualitative analysis of responses served to classify the participants in levels of performance (0-3) in each descriptor, according to the completeness of their response. In the paper we describe these levels and present examples of application for the different components analyzed by the participants. This qualitative analysis is complemented by quantitative information of the percentage of participants achieving the different levels of performance in each descriptor and the average level achieved in the group of prospective teachers.

Results suggest better performance as regards cognitive suitability than affective suitability and suggest specific points where we need to improve the knowledge of statistics and students in prospective school teachers. At the same time, the activity and the levels proposed may be used as an instrument to evaluate the knowledge of content and students in other mathematical topics. 